\title{
Autoguider locked on a fiber input for precision stellar radial velocities $^{\star}$
}

\author{
F. Bouchy and P. Connes \\ Service d'Aéronomie du CNRS, BP. 3, F-91371 Verrières-le-Buisson Cedex, France \\ e-mail: bouchy@aerov.jussieu.fr
}

Received October 23; accepted December 7, 1998

\begin{abstract}
Measurement of stellar radial velocities (RV) is one acknowledged approach for planetary-search and asteroseismology programs. We study the incomplete scrambling action of a fiber feeding a spectrograph, which leaves RV errors at a level of few $\mathrm{m} / \mathrm{s}$. Observations realised with the ELODIE fiber-fed crossed-dispersion spectrograph at the 193-cm telescope of Observatoire de Haute-Provence (OHP) are presented. A fiber-locked autoguider (called FLAG) has been specifically built to reduce the stellarbeam geometrical fluctuations within the spectrograph. With FLAG, the fiber-input plays the role of guiding detector. Automatic focusing is also accomplished by the system. The design and performance characteristics of the instrument tested at the $152-\mathrm{cm}$ telescope of OHP are reviewed here.
\end{abstract}

Key words: atmospheric effects - instrumentation: spectrographs — techniques: radial velocities — stars: oscillations — planetary systems

\section{Introduction}

We consider here planetary-search or asteroseismology programs. With CCD-equipped crossed-dispersion spectrographs, photon noise permits in principle to measure the radial velocity (RV) changes of many stars using relatively small telescopes with a precision of the order of $1 \mathrm{~m} / \mathrm{s}$ (Connes 1985; Brown 1990). In order to achieve this precision in practice, steps must be taken to control many sources of instrumental errors. A major one is due to the motions of the star image across the spectrometer input due to telescope-guiding or seeing fluctuations. The slit width in a high-resolution echelle spectrograph, in RV units, is typically a few $\mathrm{km} \mathrm{s}^{-1}$. Thus, if the photocenter

\footnotetext{
Send offprint requests to: F. Bouchy

* Based on observations collected at the Observatoire de Haute-Provence, CNRS, France.
}

of the star image suffers a shift equal to $10^{-3}$ slit width, errors larger than $1 \mathrm{~m} / \mathrm{s}$ will occur. Since the slit width projected on the sky is typically a few arcsec, it seems that milli-arcsec guiding precision is required. One very successful method makes use of a molecular absorption cell to impress lines of stable wavelength on the incoming starlight (Butler et al. 1996; Cochran \& Hatzes 1994; Walker et al. 1995). The stellar and reference spectra are then recorded from the same beam, thereby circumventing the problem of the photocenter shift. However this approach increases considerably the photon-noise RV errors, hence requires larger telescopes for a given star. Another method, which avoids this drawback, makes use of a fiberoptic feed for the starlight, plus a second fiber carrying light from a stable wavelength source (Mayor \& Queloz 1995; Brown et al. 1994). One may also use a single fiber alternately for both beams, as intended for the "absolute accelerometry" (AAA), proposed by Connes (1985) and developed by Schmitt (1997). Unfortunately, the scrambling action of a fiber reduces but does not cancel the stellar-beam geometrical fluctuations within the spectrograph. The use of a double-fiber scrambler is a current partial cure. The present paper is devoted to a study of these effects, and to the description of another partial cure: a fiber-locked auto guider (called FLAG), in which the fiber-input plays the role of guiding detector.

Several fast-guiding (or so-called "tip-tilt") systems have recently been described (e.g. ISIS, DISCO, HRCam, AOC, FASTTRAC, UH/Ifa $\left.{ }^{1}\right)$. In all cases, the goal is reduction of stellar image size as recorded over "long" exposures (i.e., seconds or minutes) through elimination of fast photocenter wandering. The overall improvement may be as large as 2, for small values of the FRIED ratio $D / r_{\mathrm{o}}$. An even greater gain is realized from adaptive optics $(\mathrm{AO})$, which eliminate not only photocenter

\footnotetext{
1 ISIS: Thompson \& Ryerson (1984), DISCO: Maaswinkel et al. (1988), HRCam: Racine \& McClure (1989), AOC: Golimowski et al. (1992), FASTTRAC: Close \& McCarthy (1994), UH/Ifa: Pickles et al. (1994).
} 
motion, but also instantaneous image blurring from wavefront distortion; this is achieved at the cost of many servo channels, hence far greater complexity.

The goal of FLAG is different. Image size reduction by itself will be welcome, as leading to either more photons in the spectrometer, or higher resolution or both. However, with meter-size telescopes in the visible, the improvement may be small at best: e.g., in our Sect. 5.1.2 tests, $D=152 \mathrm{~cm}, r_{\mathrm{o}}=6 \mathrm{~cm}$, and Hecquet \& Coupinot (1985) predict a reduction of 1.25 in image diameter. Furthermore, except for the fainter stars within the RV program, the results will not be limited by photon noise but by "scrambler noise". Hence, stabilisation of the fiberouput beam is our prime goal. Moreover, a planetarysearch program involves a large number of "exposures", stretching over years; hence, it is not for minutes but for years that we want to keep our beam stable irrespective of drifts in the autoguider, possibly even of guiding-detector changes. For the same reason, we also want to correct focus position.

A last difference: image size improvements from the above tip-tilt devices may be predicted from atmospheric turbulence models, and actual agreement is not too bad. Here, the situation is far more complex: the beam is fed not to a plain imaging camera, but to a camera through a fiber first, and a spectrograph second. There is no practical way of modeling the overall optical system with the precision required here. Presently, all fiber-plus-spectrograph stellar-RV programs (including ours) are stuck at an error level of a few $\mathrm{m} / \mathrm{s}$ : this state of affairs could not have been predicted from any theoretical calculation, and still cannot be accounted for quantitatively. This situation may change drastically in the future, should AO devices progress enough to give acceptable STREHL ratios down to $400 \mathrm{~nm}$ for a 1 or $2-\mathrm{m}$ telescope. As pointed out in Sect. 2, we may then feed the spectrometer with a singlemode (SM) fiber, which acts as a perfect scrambler; the output-beam (easily computable) will then be fully stabilized, irrespective of any input-beam fluctuation, and even of residual $\mathrm{AO}$ errors.

Returning to the present day, the intent and limitations of FLAG may now be better understood: Unlike the builders of the current tip-tilt systems, we cannot put a number to the expected improvement; worse, we cannot even hope to demonstrate a large one within the short time range of the observations reported here. Like them, we are doing the best we can with a simple device, which risks obsolescence from future AO progress.

A brief review of the scrambling properties of stepindex fibers and their use in astronomical spectrographs is given in Sect. 2. In Sect. 3, we present our own measurements of stability of the stellar beam within the ELODIE fiber-fed spectrograph, using an available but inadequate standard autoguider based on a CCD detector. In Sect. 4, we describe our prototype FLAG, built for the new spectrograph EMILIE (under construction, Bouchy et al. 1999), and the results are discussed in Sect. 5. The system is fully compatible with a double-scrambler, but has not yet been used with one.

\section{Scrambling properties of fiber}

A review of fibers in astronomy has been given by Heacox \& Connes (1992). Fiber-fed spectrographs use multi-mode (MM) step-index fibers with core sizes typically in the 50 - to $500-\mu \mathrm{m}$ range. One property of fibers, discussed by Ramsey (1988) and Barden (1995), is focal ratio degradation (FRD). A fiber tends to increase the divergence (or "speed") of the beam it carries. This causes either a loss of light if the exiting cone of light overfills the spectrograph collimator, or a loss of resolution if the collimator is replaced with one matching the faster focal ratio. Largercore fibers do appear to have better FRD characteristics than smaller-core ones. Faster input focal ratios are better preserved than slower ones. One other significant characteristic of fiber optics is their ability to scramble the input image. The geometry of cylindrical fibers introduces two dimensions of scrambling, azimuthal and radial. Both theory (Heacox 1987) and experiments (Hunter \& Ramsey 1992) suggest that step index MM fibers provide a high degree of azimuthal scrambling but an incomplete radial scrambling. Image scrambling improves with an increase of the fiber FRD. If the input focal-ratio is fast enough to give little FRD (and this is the case in fiber-fed spectrographs), then the input image will be poorly scrambled, and the output beam preserves some memory of the input beam shape and position.

Our laboratory tests on a $50 \mu \mathrm{m}$ step index MM fiber, and also tests by Casse (1995) on a $300 \mu \mathrm{m}$ fiber, both measure the photocenter of the near-field versus a known displacement of the input beam. We found independently that the motion of the output photocenter may be of the order of 100 times smaller than that of the input photocenter, with adequate beam and fiber parameters.

This imperfect scrambling is inadequate when the goal is $1 \mathrm{~m} / \mathrm{s}$ precision. The near-field pattern of a fiber is defined as the brightness distribution across the output face of the fiber. In most cases a spectrograph images this output face directly onto the detector; then variations in illumination introduce small changes in pixels illumination which lead to radial velocity shifts. A higher-resolution spectrograph reduces these errors, because given image shifts on the pixels induce smaller wavelength shifts than in a lower-resolution spectrograph.

The far-field pattern is defined as the cross-section of the beam far from the output face. Far-field variations are projected onto the spectrograph collimator, and cause changes in grating illumination. Then, spectrograph aberrations and grating imperfections induce small varying RV shifts. To increase scrambling, one may incorporate a double-fiber scrambler (Brown 1990), in which a pair of 
fibers is coupled together using a pair of microlenses, separated by their common focal lengths. The fibers then see each other at infinity, causing the near- and far- fields to be interchanged. Brown et al. (1991) found that their doublescrambler eliminated temporal variations in grating illumination that were responsible for roughly $5 \mathrm{~m} / \mathrm{s}$ RMS velocity noise in their spectrograph and increased the precision of radial-velocity observations by a factor of about 3 over a single fiber, to the expected shot-noise-limit. The same double scrambler was laboratory-tested by Hunter \& Ramsey (1992). Their results show that intensity variations across the near- and far-field patterns due to both angular and positional changes in the input beam were reduced by a factor ranging from 2 to 10 . Casse (1995) laboratory-tested a similar double-scrambler and found a scrambling gain of about 5 in the near-field pattern. The decreased radial-velocity noise provided by these doublescramblers is not without drawbacks: the main problem is low throughput. The transmission estimated both by Hunter \& Ramsey (1992) and Casse (1995) is between 20 and 25\%; Brown (1994) mentioned 66\%. A somewhat different double-scrambler was incorporated in March 1997 at the input of the fiber-fed ELODIE spectrograph by D. Kohler. Its throughput is about $75 \%$ but the overall gain in radial-velocity precision deduced from our own observations is only about 1.5. According to Mayor and Queloz (private communication) the current long-term precision limit of ELODIE is now not due to the scrambler but related to the thermal and mechanical variations of the instrument. Probably their relative long exposures (greater than 10 minutes) averages the scrambler noise. In our case, with time exposure less or equal to 1 minute, we are not convinced that a double-scrambler is sufficient.

Another solution (proposed by Connes et al. 1996), will cancel "scrambler noise" altogether. It makes use of a single-mode (SM) fiber which (unlike a MM fiber) acts as a perfect SM spatial filter: all cross-sections of the output beam are quasi-Gaussian and preserve no memory of the input beam geometry. Hence a SM fiber behaves as an ideal scrambler. It may be matched to the Airy pattern at the focus of a diffraction-limited telescope, irrespective of its diameter, with an efficiency of about $80 \%$. On the ground, this solution is limited to a telescope pupil smaller than the "Fried diameter" (roughly $12 \mathrm{~cm}$ in the visible for 1 arcsec seeing), or requires AO. Such systems are so far limited to the infrared. Altogether, while the association of an SM fiber and AO offers a definitive solution of our problem, it cannot be tried presently.

\section{Fluctuations of the stellar beam at the output of the fiber feeding the ELODIE spectrograph}

\subsection{ELODIE spectrograph}

A full description of ELODIE is given by Baranne et al. (1996). This is a fiber-fed spectrograph within a stable temperature-controlled environment, located at the

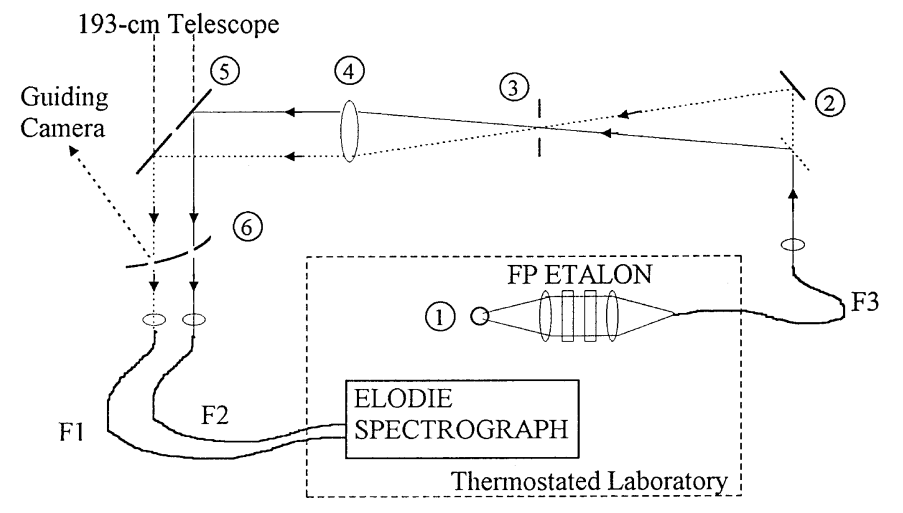

Fig. 1. ELODIE Calibration optical system: 1) tungsten lamp, 2) mirror and beam splitter, 3) pupil, 4) achromat (to put the pupil at infinity), 5) removable mirrors, 6) concave mirror with 2 holes. Fiber F3 takes FP beam to Cassegrain focus assembly (here highly schematized; for more exact diagram, see Fig. 4 of Baranne et al. 1996). Fibers F1, F2 are illuminated through holes in mirror 6. Mobile mirror system gives two possibilities: 1) FP light into $\mathrm{F} 1$ and $\mathrm{F} 2$; 2) star light into F1, FP light into F2. This system is standard; we added only the FP étalon

193-cm telescope of Observatoire de Haute-Provence, in France. Using a $102 \times 408 \mathrm{~mm}$ echelle and a $1024 \times 1024$ CCD, it samples a spectrum between 389 and $681 \mathrm{~nm}(67$ echelle orders) with a spectral resolution of about 42000 . The pixel velocity-width is about $3000 \mathrm{~m} / \mathrm{s}$. The $100 \mu \mathrm{m}-$ core fiber input accepts 2 arcsec from the sky. The beam aperture is converted to $\mathrm{f} / 5$ by transfer optics in order to feed the fiber, located at the $\mathrm{f} / 15$ Cassegrain focus, and then brought back to $\mathrm{f} / 15$ at the spectrograph input. At the focus of the $\mathrm{f} / 3$ camera lens, the geometrical spot diameter is $60 \mu \mathrm{m}$ corresponding to a velocity-width of about $7500 \mathrm{~m} / \mathrm{s}$. It is feasible to record simultaneously star and reference spectra with two fibers, the outputs of which are displaced by few pixels in the direction of cross dispersion, and orders for both spectra alternate over the CCD. For our observations, we introduce the channelled spectrum from a Fabry-Perot (FP) etalon illuminated by a white-light source (Fig. 1). With invar spacers and temperature stabilization within $10^{-2} \mathrm{~K}$, the FP-induced velocity error is about $3 \mathrm{~m} / \mathrm{s}$ but appears only as a slow drift during a run. Two kinds of recordings are possible: FP/FP spectra (with the FP beam on both fibers) and STAR/FP spectra. We record simultaneously the two spectra, extract the echelle orders, and compute the RV change relative to a reference exposure. In order to get meaningful RMS errors within a few hours, and also to check the suitability of ELODIE for studying stellar oscillations, we used a large number (a few hundreds) of short exposures $(60 \mathrm{~s})$ on a given star. A full description of our RV measurements with ELODIE has been given by Connes et al. (1996).

The autoguider is the common-user 193-cm-telescope device, the principle of which is standard: a tilted concave mirror pierced with a hole corresponding to 


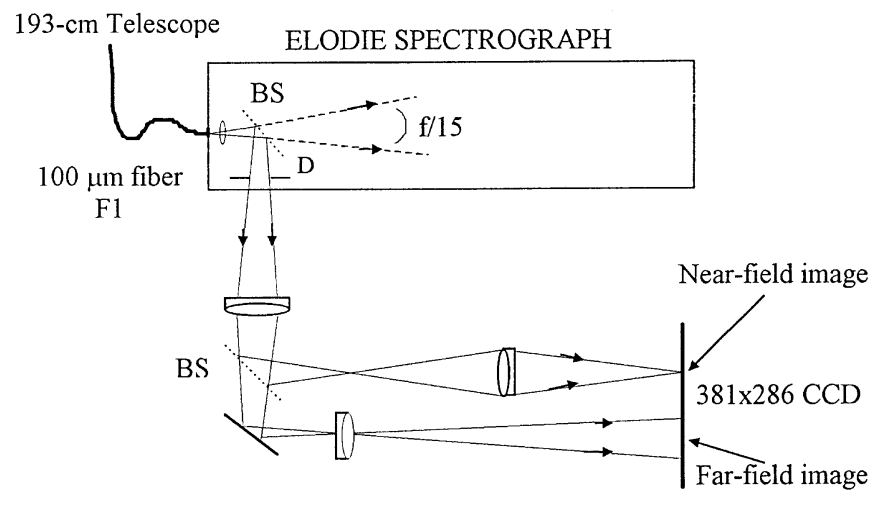

Fig. 2. Fiber-output optical section (added by ourselves) for measurement of beam geometrical fluctuations at spectrograph input (simplified diagram, not to scale). System is located on an aluminium table suspended below spectrograph, and fed through hole in granite baseplate. The Kohler doublescrambler was not yet available

2 arcsec on the sky allows the fiber to be fed and, at same time, the outer part of the image to be reflected onto the VIDICON guiding camera (see Fig. 1). Initial image centering is done manually from the video-screen image; then the autoguider is locked on. An HP1000 computer calculates the coordinates of image centroid and triggers relays which operate the fine-guiding telescope motors (with step unit 0.1 arcsec) in order to return the image to the center position. In general, an average of many video images is used before making a correction, which reduces by integration the guiding-camera noise, smooths out the effect of seeing, and damps the telescope response. Corrections are performed once every few seconds, which means (in our case) at least 10 corrections per spectrograph exposure. The system does not measure seeing.

\subsection{Observations and data reduction}

In a previous study, Connes et al. (1996) have measured apparent fluctuations in stellar velocities with ELODIE. For bright stars, these were much higher than the photon noise. This effect did not appear when a spatially stable reference beam was used instead the star. An imperfect scrambling of the stellar beam ("scrambler noise") was obvious. Here, we attempted to quantify the geometrical time-fluctuations of the output beam of the stellar fiber. For this experiment, about $30 \%$ of the output beam was taken with a beam-splitter located within the spectrograph, and the near- and far-fields of the stellar fiber output were re-imaged on the same $381 \times 286$ CCD. A sketch of the optics is shown in Fig. 2, and a typical image in Fig. 3.

In order to detect unusual problems in time to correct them during the observing run, an on-line data treatment was applied. Images were reduced directly after each exposure, and also saved on a PC disk for future analysis.
The program computed for each field (limited to a specific window on the CCD) the $X Y$ photocenter, the first order momentum and the intensity average within the window. The differences between these parameters and those computed from a reference image taken at the beginning of the run were displayed on-line. Fluctuations are presented in nanometers for the near-field, in microradians for the far-field; relative changes are also given. We estimate that our system could measure CG motions of about 1/1000 pixel, which means about $1 \mathrm{~nm}$ on the output face of the $100 \mu$ m-fiber.

Acquisition of each field pattern was synchronous with spectrograph exposures, in order to compare the different image parameters with the RV measurements.

\subsection{Results}

\subsubsection{Fabry-Perot étalon beam}

Our first experiment used the white-light source and FP étalon only. Hence, the beam falling on the fiber input was geometrically stable during a run. We took simultaneous 62 seconds exposures with the spectrograph $1 \mathrm{kx} 1 \mathrm{k}-\mathrm{CCD}$ detector and with our Fig. $2381 \times 286$-CCD detector. The lost time from readout plus computation was $40 \mathrm{~s}$, hence the cycle time was $100 \mathrm{~s}$ and the sequence took about 5 hours. Figure 4 a presents the $X, Y$ near-field photocenter shifts in nanometers versus time. The slow drift is attributed to mechanical relaxations and temperature drifts mostly within the Fig. 2 optical system, hence is uninteresting. We fit our data with a 3rd-order polynomial and find a fluctuation of $5 \mathrm{~nm}$ RMS, roughly explainable by photon noise. Figure $4 \mathrm{~b}$ presents the difference between the two spurious-RV curves, obtained with FP light on both fibers, relative to a previously exposed reference spectrum and computed from a single echelle order (centered at $571 \mathrm{~nm}$, and covering $6.1 \mathrm{~nm}$ ). Such a test is of course independent of any FP drift. The slow trend shows that the two-fiber setup does not compensate fully spectrograph drift (as already shown by Connes et al. 1996). The residual may arise from a nanometer-scale relative drift between the two fibers outputs; it may also involve data treatment and non-identical sampling of the two spectra by the CCD since the absolute drift (for both fibers) was about 100 times larger (see Fig. 3 of Connes et al. 1996). The best cure should be alternate use of the same fiber for both beams. The fast residuals $(0.82 \mathrm{~m} / \mathrm{s}$ RMS) come mostly from photon noise, as shown by the fact that they decrease as expected when the RV from many orders are averaged.

\subsubsection{Stellar beam}

We now have star light on fiber F2 and FP light on F1. The star $\gamma$ DRA $\left(M_{v}=2.2, \mathrm{~K} 5 \mathrm{III}\right)$ was selected because 


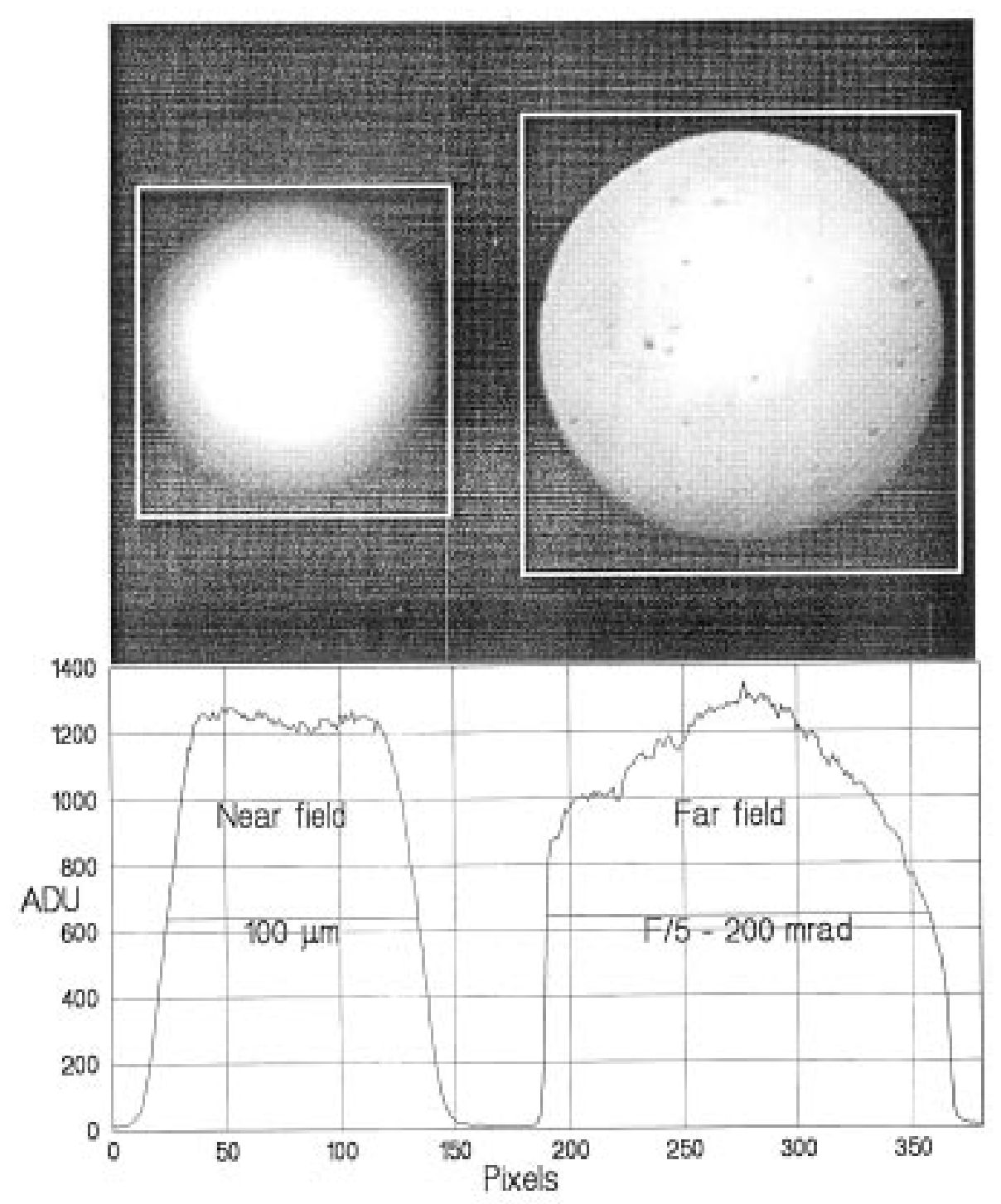

Fig. 3. Fiber-output beam cross-section from a star (193-cm telescope, $100 \mathrm{~mm}$ fiber). Near-field: left; far-field: right. Two windows $(140 \times 140$ pixels and $190 \times 190$ pixels $)$ used for computation are shown. Pixel size: $23 \mu$ m; dynamic range: 12 bits. $100 \mu \mathrm{m}$ and $\mathrm{f} / 5$ indicated dimensions are reduced to fiber output. A small portion of the far-field is cut-off on the left side by a holder within the spectrograph

it could be observed all night. A sequence of 130 similar 62-s exposures with 111-s cycles was recorded. Figure 5a presents near-field $X$ and $Y$ photocenter shifts, Fig. 5b the radial velocity (from a single order, after subtraction of 2nd order polynomial, as only fast fluctuations are of interest here), Fig. 5c the mean intensity for the same order, plus star elevation. The seeing could not be monitored continuously, but did not seem to depart greatly from the usual OHP figure of 2 to 2.5 arcsec.

Two main remarks: 1) Missing data points correspond to electronic failures of the Fig. 1 removable mirror system; they are unrelated to guiding problems. 2) Even with the guider ON, the stellar image was invariably seen to drift relative to fiber input on the video screen, which indicates poor stability of something in the guider op- tics. Whenever this drift seemed excessive (i.e. approaching image size), the guider was stopped for few seconds, and the guiding point recentered, again from the video image. Major peaks (marked by arrows) indicate these operations.

These irregular and irreproductible guider incidents contribute a major part of the measured RMS, both for $X$, $Y$ and for the RV. Here we get $9.26 \mathrm{~m} / \mathrm{s}$; in our older tests (Connes et al. 1996), with similar seeing, we had found $6.2 \mathrm{~m} / \mathrm{s}$.

Lastly, although a large part of the intensity fluctuations arose from changes in seeing and/or transparency, some peaks are correlated with those in Fig. 5a while the reduction of intensity is minimal. Hence, guiding errors not only decrease the intensity of the stellar beam 

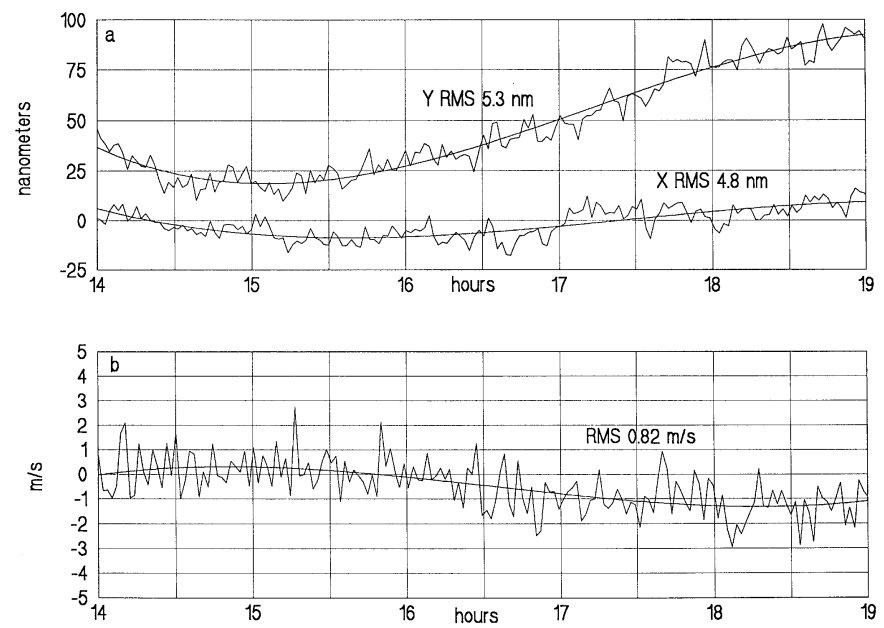

Fig. 4. Output beam fluctuations from FP beam. a) Near-field photocenter $X$ and $Y$ shifts measured by Fig. 2 set-up. b) RV measured by ELODIE

but also change the geometry of the beam in the spectrograph, and degrade the RV results. If we suppose that the $100-\mu \mathrm{m}$ fiber reduces the star image motions by a factor 100 (as in Sect. 2), this means that these motions are about $5 \mu \mathrm{m}$ RMS, which corresponds to 0.1 arcsec; this is the step-size of the fine-guiding telescope motors.

\subsubsection{More guiding problems}

Two other severe drawbacks of the 193-cm telescope autoguider for our program were also noted:

First, the autoguider does not check nor correct focusing. The size of the video image is seen to drift, and manual focus corrections produce similar RV breaks.

Second, this autoguider fails to work with alternate illumination of fiber F1 by the star- and FP-beams; operation of the Fig. 1 removable mirrors is not possible within a sequence such as those of Figs. 4 or 5. We have seen that mere use of the second fiber F2 for the reference beam leaves uncorrected errors of a few $\mathrm{m} / \mathrm{s}$.

As far as a search for stellar oscillations during a given night is involved, these problems are likely to find simple solutions. For instance, no obnoxious guider drift is mentioned by Brown et al. (1994), whose RV records also cover a few hours. Our 193-cm telescope has a Pyrex-type mirror; with a ZERODUR one, short-term defocusing might become negligible. However, irrespective of any guider improvements, similar difficulties are bound to arise during a long-term planetary search. For instance, the VIDICON will soon be replaced by an intensified CCD; this will mean a RV break. So far, within any very-long-term program, stellar image centering and focusing are performed from qualitative eye-only estimates.

The AAA technique involves use of a wavelengthsliding reference spectrum, reducing the spectrograph role
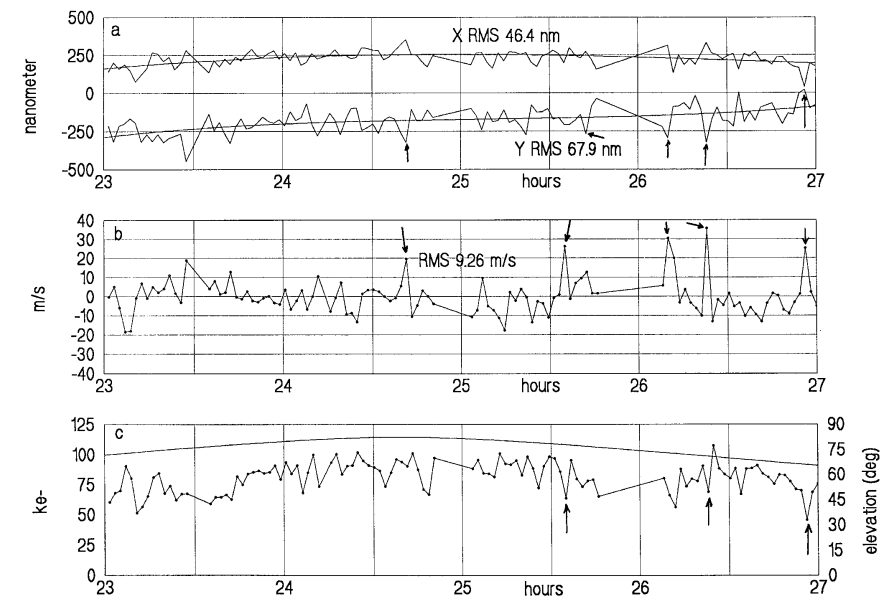

Fig. 5. Output beam fluctuations with star. a) Near-field photocenter $X$ and $Y$ shifts. b) RV measured by ELODIE and corrected of the Earth motion. c) Intensity fluctuations and star elevation

to the classical one of a null-checking device. It eliminates all internal spectrograph problems; even a CCD replacement at spectrograph output should become irrelevant. However, a geometrically-stable stellar beam, alternating with the reference beam, is still essential at the spectrograph input. In order to guarantee that performance over the long term (several years), a sizeable effort was clearly required to solve the guiding problem. Altogether, an autoguider making use of the fiber input itself as a nullchecker of stellar-image $X Y Z$-position appeared best, as it should solve guiding and focusing difficulties simultaneously. Such a system is now to be described.

\section{Fiber locked auto guider (FLAG)}

The goal of FLAG is to cancel at fiber output all effects arising from stellar-image photocenter $X Y Z$ motions. Admittedly nothing is done about instantaneous image blurring, which should then become responsible for any remaining perturbations.

\subsection{Principle}

The fiber input itself plays the role of position detector. One introduces two small oscillations of stellar image in two perpendicular directions $X$ and $Y$, with frequencies $F_{x}$ and $F_{y}$. A small translation of a lens on its axis produces a $Z$-translation of the image, hence a focusing change with frequency $F_{z}$. At the output of the fiber, any type of single-pixel intensity detector generates a modulated signal; it uses the NIR through a dichroïc beamsplitter. Three synchronous demodulators at frequencies $F_{x}, F_{y}$ and $F_{z}$ reconstruct three DC error signals $S X, S Y$ and $S Z$. The first two are applied to some fast tip-tilt 


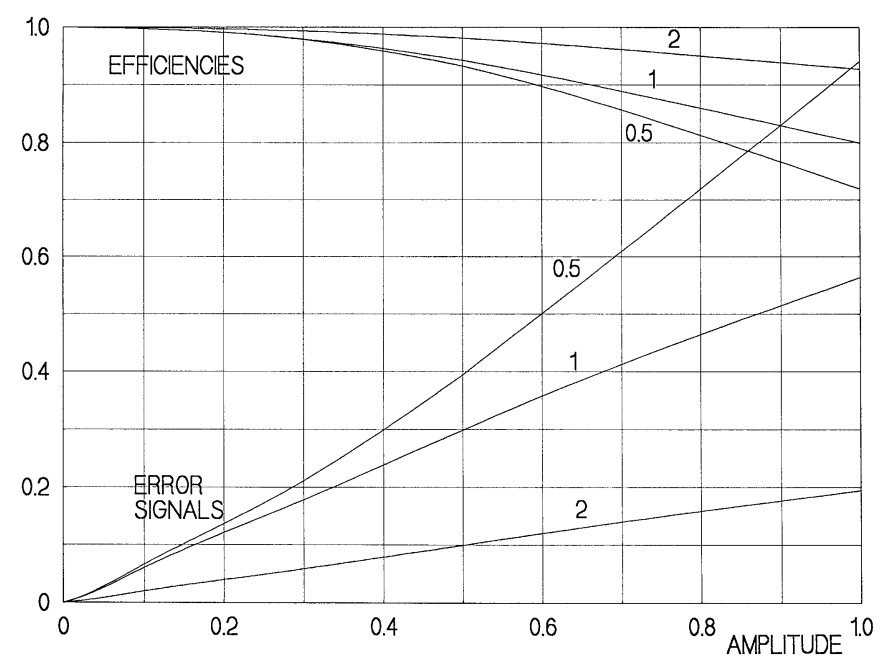

Fig. 6. FLAG basic trade-off; numerical simulation for a single channel. Growth of error-signal (arbitrary units) versus $X$ or $Y$ oscillation amplitude (unit: fiber radius), and decrease of efficiency. Three cases shown, for FWHM of Gaussian seeing pattern equal to $0.5,1$, or 2 fiber diameters. Usual compromise in our tests: amplitude 0.25, efficiency 0.98. Similar curves are found for $Z$. For all three channels active, efficiency then drops to 0.94

device, while $S Z$ may go either to the $Z$-lens, or to the telescope secondary-mirror motor.

The image oscillations widen the seeing pattern, hence induce some loss at fiber input; numerical simulation for a single $X$ or $Y$ parameter is shown by Fig. 6 ; for $Z$ the curves are similar. When all 3 channels are active together, and at least for amplitudes $\ll$ fiber radius (as used in practice), the error signals remain the same, with little crosscoupling, while the overall efficiency is the product of the individual ones. Oscillation amplitude is adjustable at will, and the optimum trade-off depends on stellar color and magnitude. In practice, we have kept constant amplitude, obtained nearly constant performance, up to $M_{v}=8$, and measured a $6 \%$ loss by switching all three oscillations on and off. The dichroïc loses another $15 \%$ of the visible beam.

There are some possible variants, all untried so far: With minor changes, a photon-counter could be substitued. A single frequency might be used for $X$ and $Y$, with $90^{\circ}$ phase difference; image trajectory would then become a circle rather than a Lissajous pattern. Instead of a dichroïc, a weak achromatic beamsplitter might bleedoff a small fraction of the visible beam. Finally, a double scrambler might be incorporated without any change of the system.

\subsection{Optical system}

Our design was intended for the Coudé focus of the 152-cm OHP telescope (Fig. 7); furthermore, it made use

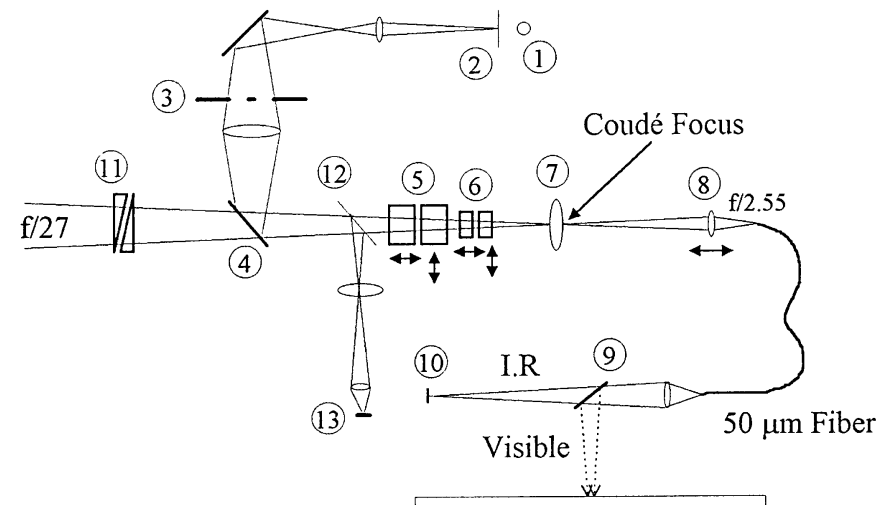

Fig. 2 Optical System

Fig. 7. Autoguider optics: 1) tungsten lamp, 2) circular Gaussian-pattern screen, 3) simulated telescope-pupil stop, 4) commutation mirror, 5) guiding plates $(10 \times 10 \times 20 \mathrm{~mm}), 6)$ scanning plates $(5 \times 5 \times 5 \mathrm{~mm})$, 7) field lens, 8) translating achromat, 9) dichroïc, 10) guiding detector, 11) atmospheric dispersion compensator, 12) beamsplitter or removable mirror, 13) finder video. Commutation mirror is removed for star beam

of available or inexpensive elements only. The $\mathrm{f} / 27$ beam is converted to $\mathrm{f} / 2.55$, and the star image is formed on the $50 \mu \mathrm{m}$-fiber entrance which accepts 2.7 arcsec on the sky. The two plane-parallel "scanning" plates (6), close to the Coudé focus, introduce two small oscillations of the image $(\sim 0.2$ arcsec $)$ in two perpendicular directions $X$ and $Y$, with frequencies $F_{x}(1190 \mathrm{~Hz})$ and $F_{y}(955 \mathrm{~Hz})$. The larger "guiding" plates (5) can introduce a tilt up to \pm 4 arcsec. The field lens (7) (focal length $70 \mathrm{~mm}$ ) reimages the telescope pupil on the achromat (8) which is mounted on a small loudspeaker (drilled through its axis) and oscillates in $Z$ at frequency $F_{z}(215 \mathrm{~Hz})$. The very short 6-mm focal length makes chromatic aberration negligible, which is important as guiding and focusing are checked in the NIR.

At the fiber output, the beam is split by a $600-\mathrm{nm}$ cutoff dichroïc plate (9). The NIR part falls on a Si avalanche diode, Peltier-cooled (10). In order to make direct measurements of beam shape and position, the visible part produces images of the near- and far-field as in Fig. 2. Later on, this same beam will be sent to the EMILIE spectrograph (Bouchy et al. 1999). An atmospheric-dispersion compensator (11) incorporates a pair of counter-rotating low-dispersion prisms piloted by a PC; it is independent of the guider. A finder (12-13) is used to center the star image on the fiber $(5 \times 7 \mathrm{~mm}$ CCD video camera with a $2 \times 3$ arcmin field of view). The commutation mirror (4) interchanges stellar and reference beams. A set of Gaussian screens (2), illuminated by a tungsten lamp (1), simulates stars under different condition of seeing. An aperture stop (3) with a central obstruction, is located in a plane 


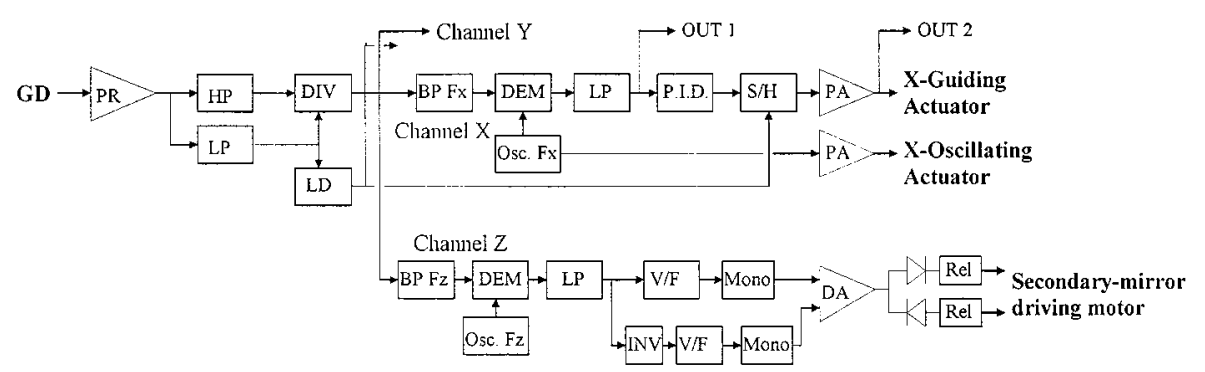

Fig. 8. Autoguider electronics. GD: guiding detector, PR: preamplifier, HP: high pass filter, LP: low pass filter, DIV: analog divider, BP: band pass filter, DEM: demodulator, PID: proportional-integrator-derivator, S/H: sample/hold, PA: power amplifier, OSC: oscillator, V/F: voltage-to-frequency converter, MONO: monostable multivibrator, INV: inverter, DA: differential amplifier, REL: relays

conjugate of the telescope pupil; hence the beam shape matches that from the telescope.

\subsection{Electronic control system}

The system is controlled by purely analog electronics (Fig. 8); it was built without outside help, entirely on solderless test boards. Let us follow the $X$-guiding channel. An harmonic oscillator signal at frequency $F_{x}$ is applied to the $X$-actuator. If the image mean position is not centered on the fiber input, the guiding detector signal GDS is modulated at $F_{x}$. For exact centering, the $F_{x}$ fundamental must be nulled, and only harmonic 2 remains; hence, a demodulator at $F_{x}$ provides a DC error signal, and a proportional-integrator-derivator (PID) optimizes the servo response. The oscillation actuators have $1.5 \mathrm{kHz}$ resonant frequencies, the guiding ones about $200 \mathrm{~Hz}$. A first version of the system used only 2 plates, both for scanning and guiding, with the slower actuators; it operated correctly, but was slower.

For the $Z$-channel, the oscillator signal $F_{z}$ goes to the $Z$-lens loudspeaker. The DC correction can be applied either to the loudspeaker or to the telescope secondarymirror driving motor. We tried both schemes; with the loudspeaker, $Z$-correction was fast but perturbations appeared on $X$ - and $Y$-channels, probably due to poor optical alignment. Since telescope defocusing is very slow, the second scheme (shown in Fig. 8) gave adequate results. Two V/F converters provide slow pulse trains (one pulse for every few seconds); two monostable multivibrators adjust the length of the motor-on intervals, hence correction speed. The system is roughly equivalent to the Integrate channel of the PID.

The rest-point of all three servos is independent of the mean GDS intensity, but the response time is not, and they would become sluggish when the beam passes through weak clouds. Hence an automatic gain-corrector is added at preamplifier output. An analog divider provides the ratio of $\mathrm{AC}$ to $\mathrm{DC}$ components; the output is a stabilized-amplitude AC signal, and is fed to the demodulators. The system works well within a 10/1 range of
GDS intensity, greater than needed for recording spectra in practice.

During short but total cloud-induced breaks, recording has to stop, and it does not matter that the error signal is no longer available. However, when the star reappears, one wants the autoguider at least to pick-up the image automatically. A level detector triggers at $1 / 10$ th of the maximum GDS intensity, and commands a sample/hold amplifier to preserve the power amplifier input signal during the break. Thus, the autoguider is able to find the image at the point of the field where it was last seen.

Analog $X-Y$ displays of either the error signals or the guiding plate tilts are shown on an oscilloscope screen. This allows the observer to monitor the behavior of the system and to correct telescope pointing when the image drift has exceeded the guider-correction field. Later on, we may automatize the process by sending the very low-frequency guiding errors directly to the fine-guiding telescope motors.

\section{Tests and results}

Since the EMILIE spectrograph is not yet available, these tests cover only the beam-handling of FLAG; no radial velocities have been measured so far.

\subsection{Autoguider performance}

\subsubsection{Laboratory tests}

Using the tungsten lamp and a 2 arcsec FWHM Gaussian pattern, we tested each channel by introducing a known step-type perturbation. Figure 9 shows the response of channel $X$ to an 0.8 arsec step function, for one particular PID adjustment. The image is re-centered in $\sim 15 \mathrm{~ms}$ and fully stabilized in $\sim 30 \mathrm{~ms}$. The noise of this channel is estimated to 0.03 arcsec RMS. Altogether, FLAG qualifies as a moderately-fast autoguider. 


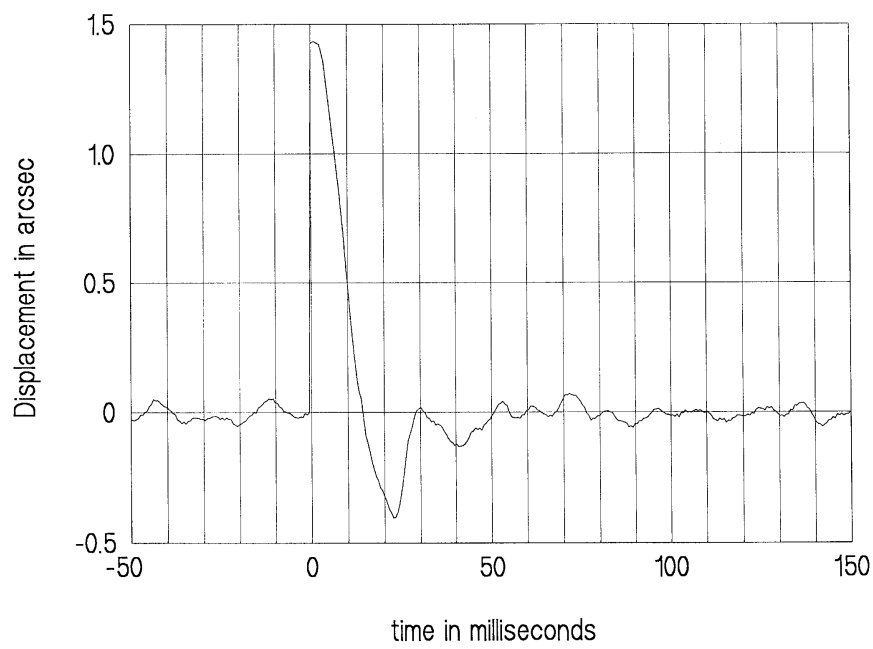

Fig. 9. Step response. Perturbation: $\sim 0.8 \operatorname{arcsec}$ on $X$-channel only

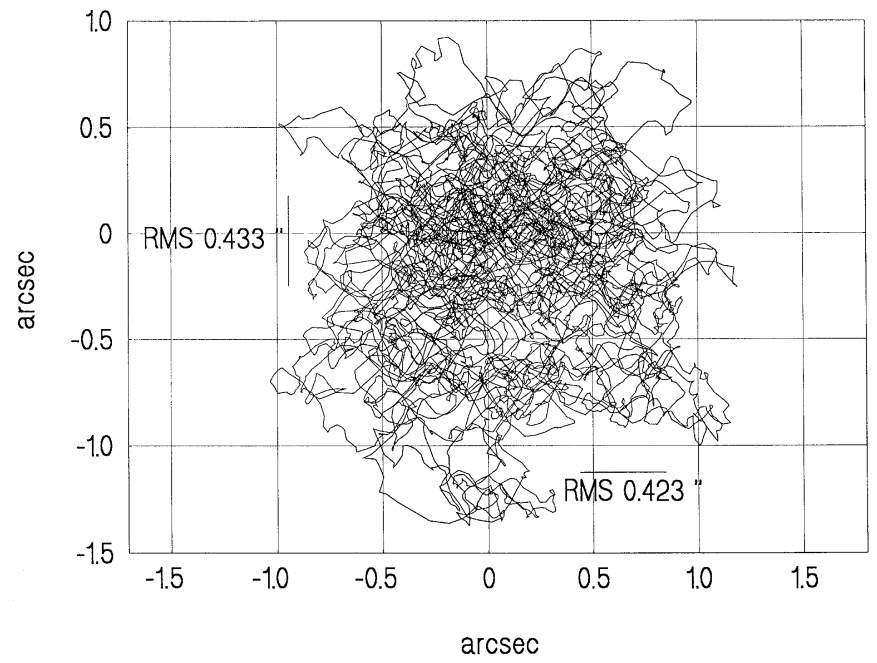

Fig. 10. $X Y$ corrections which reproduce seeing-induced photocenter motions. 2.2 arcsec seeing, total time $5 \mathrm{~s}$, star $\alpha \mathrm{UMA}$, elevation $70^{\circ}$. Signals from output 2 in Fig. 8. Recording by computer with $1.5 \mathrm{~ms} \mathrm{RC}$ time constant, $0.6 \mathrm{~ms}$ sampling interval

\subsubsection{Sky tests}

Figure 10 shows the angular corrections in $X$ and $Y$ of the "guiding" plates during a 5-seconds run on $\alpha$ UMA. The average seeing for this night was 2.2 arcsec (measured with a CCD at another telescope). These corrections are almost equal to the before-correction image motions, except for the small residuals discussed below in Fig. 12. The power spectral density (PSD) of image motion along $X$ axis is represented in Fig. 11. The dashed line shows the $f^{-2 / 3}$ power law for Kolmogorov turbulence (Martin 1987). One can see a spike around $8.5 \mathrm{~Hz}$ : this is a telescope resonance. Above $60 \mathrm{~Hz}$, the curve drops to the system noise. Our autoguider seems to respond correctly up to $60 \mathrm{~Hz}$.

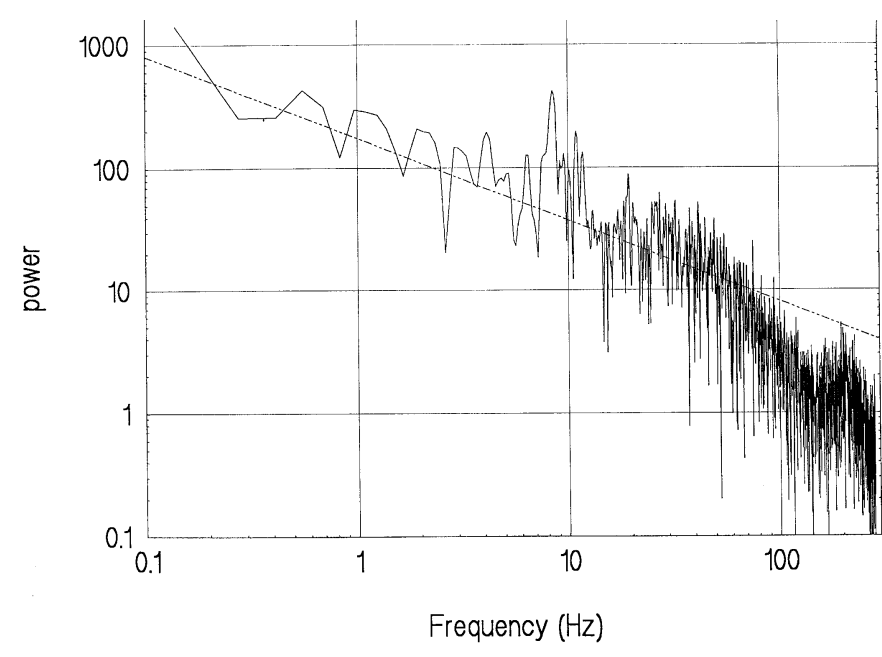

Fig. 11. Image motion spectral power density. Same data as Fig. 10. Dashed line shows slope expected for Kolmogorov turbulence

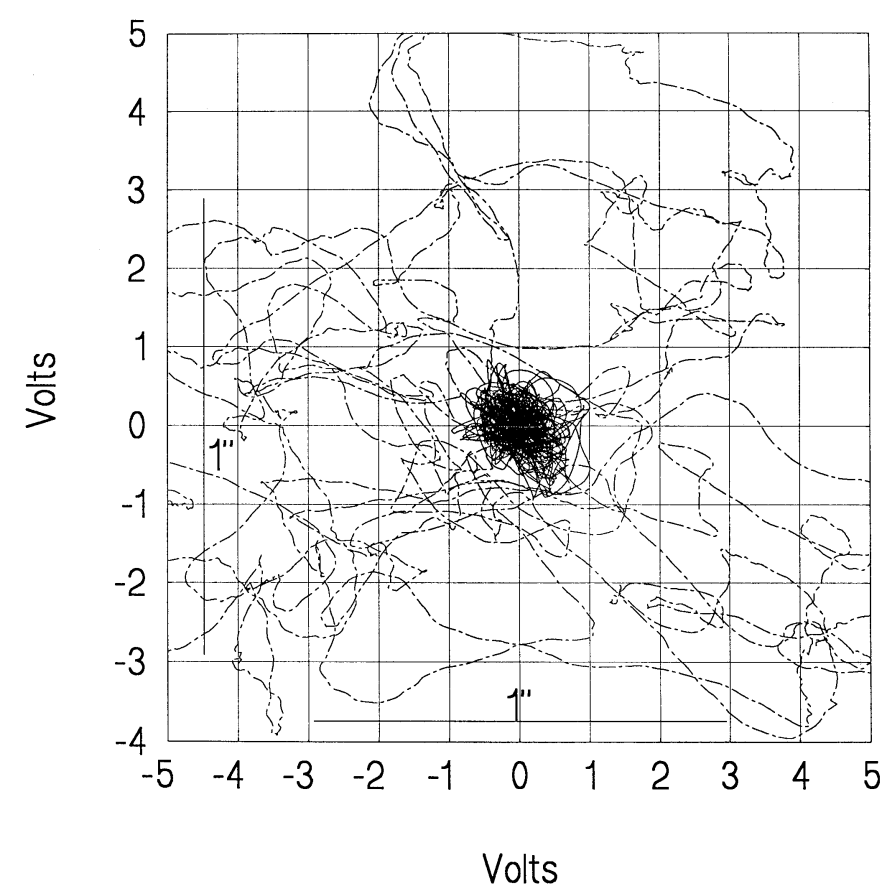

Fig. 12. $X Y$ error signals. Same conditions as Fig. 10 but from Output 1 in Fig. 8. With FLAG correction (full curve filling central "knot"). Without FLAG correction(dashed curve)

In order to show the residual (after-correction) image motion, Fig. 12 gives the error signals in volts for the $X$ - and $Y$-channel, again for 5 seconds on $\alpha \mathrm{UMA}$, with and without FLAG. One minor drawback of our technique is the absence of any straightforward conversion of error signals to angular errors. While the centering point (for which the error signals are nulled) is stable and independent of seeing, the magnitude of these signals is not. However, let us assume the seeing remained 
stable from Fig. 10 to Fig. 12. Then, the large uncorrected fluctuation of Fig. 12 (with FLAG off) is statistically the same as the FLAG correction in Fig. 10. Identifying the RMS figures, we find that in Fig. 12 a 1 volt error signal corresponded to $\sim 0.17$ arcsec for this particular combination of seeing and oscillation amplitude. From which the RMS residual photocenter motion with FLAG active (Fig. 12 central "knot") is $\sim 0.05$ arcsec, which must include a small photon-noise contribution. On the other hand, a small component of photocenter motion above the system bandpass exists, but does not show on our curves.

The system worked without appreciable degradation up to an F8, $M_{v}=8.2$ star, which is sufficient for our program of radial velocities. This limit actually came from the finder video camera. The limited tilt range of "guiding" plates ( \pm 4 arcsec) requires observer intervention 2 or 3 times per hour to cancel telescope drift; but these hand-made corrections produce no perturbation of error signals.

Most regrettably, no $Z$-error-signal recordings were made. However the $Z$-servo pulled back the telescope focus to the correct position after any manual step-perturbation. No residual image-size drift appeared on the finder video screen during full-night sequences. As expected from any noisy error signal, positive and negative corrections alternated randomly, the average interval was adjusted to roughly 10 seconds. In brief, the $Z$-channel mimics a wellbehaved but slow autoguider.

\subsection{Beam fluctuations at fiber output}

The measuring system was the one already used for ELODIE (see Fig. 2) with minor changes due to the different fiber. Figure 13 shows a typical result, on $\alpha$ UMA.

The quasi-Gaussian far-field is affected by telescope central obstruction, more so than in Fig. 3, which seems to indicate less FRD. The quasi-flat near field is affected by a narrow axial low-index zone in our $50 \mu \mathrm{m}$ fiber (FG 050 GLA from 3M). Rays are rejected from this zone by total reflection, but no loss is induced.

Our original plan had been to compare FLAG performance to that of the common-user AURELIEspectrograph CCD-guider (similar to the ELODIE one); unfortunately AURELIE had to be taken away before our observing run. Hence we had to rely on a second-best comparison, between FLAG and merely-manual guiding. FLAG was turned ON or OFF for stretches of a few minutes; when OFF, an operator kept the video star image centered through the telescope controls in the usual way, which at least removed telescope drift. The average interval between corrections was in the $5-20$ seconds range, i.e. comparable to those of the automatic ELODIE device used for Fig. 5. The main difference with the CCD has not been in speed, but in somewhat-subjective centering. A 2-hour sequence of 91 short exposures (60 s), with
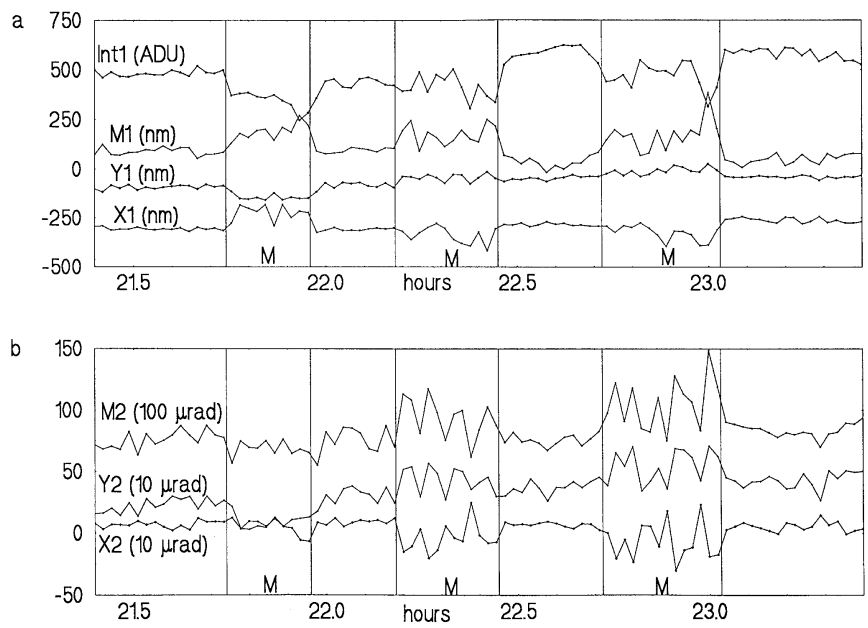

Fig. 14. a) Near- field geometrical fluctuations. INT1: image intensity in ADU. $M_{1}$ : first order momentum in nm. $X_{1}, Y_{1}$ : photocenter motion in $\mathrm{nm}$. b) Far-field geometrical fluctuations. $M_{2}$ : first order momentum in $100 \mu \mathrm{rad}$ units. $X_{2}, Y_{2}$ : photocenter motion in 10- $\mu$ rad units. Vertical lines show limits of manual guiding intervals, marked $M$

2.2 arcsec average seeing, is shown in Fig. 14, giving the geometrical fluctuations of the near- and far-fields.

The average intensity is $\sim 23 \%$ greater with the autoguider ON. This gain agrees well with the figure given by Hecquet \& Coupinet (1985) for $D / r_{\mathrm{o}} \sim 33$. The parameter $M_{1}$ (first order momentum of the near-field) give some information about the width of the image. One sees in Fig. 14a that $M_{1}$ increases during hand-guiding intervals. This means that the star image at the fiber input is degraded and widened by seeing plus tracking errors during an exposure. On the practical side, this $23 \%$ gain does no more than compensate roughly for the FLAG losses discussed in Sect. 4.1.

Data of Fig. 14 were separated in two sets corresponding to FLAG- and hand-guiding- intervals respectively. The RMS residuals from a 3rd-order polynomial fit were computed and are presented in Table 1. FLAG reduces the RMS fluctuations by factors of 2 to 4 .

Of course, from these results, we would like to predict residual RV fluctuations given by some future spectrograph, but this is frankly difficult. Only in the case of the near-field $X_{1}, Y_{1}$ can we make a try. A $10 \mathrm{~nm}$ photocenter motion corresponds to $1 / 5000$ of the fiber diameter. With EMILIE, this diameter will be imaged on (roughly) one pixel, which has $1500 \mathrm{~m} / \mathrm{s}$ velocity width; then our $10 \mathrm{~nm}$ will induce $0.3 \mathrm{~m} / \mathrm{s}$ RV change. Unfortunately, it is impossible to make similar predictions from the remaining parameters $M_{1}, X_{2}, Y_{2}, M_{2}$, or from any others (the beam cross sections have been stored for later analysis). As stressed in Sect. 1, this would require an exceedingly accurate model of spectrometer aberrations and adjustment. Furthermore, as any minor change in spectrometer focusing etc. wrecks the prediction, one doubts the effort would 


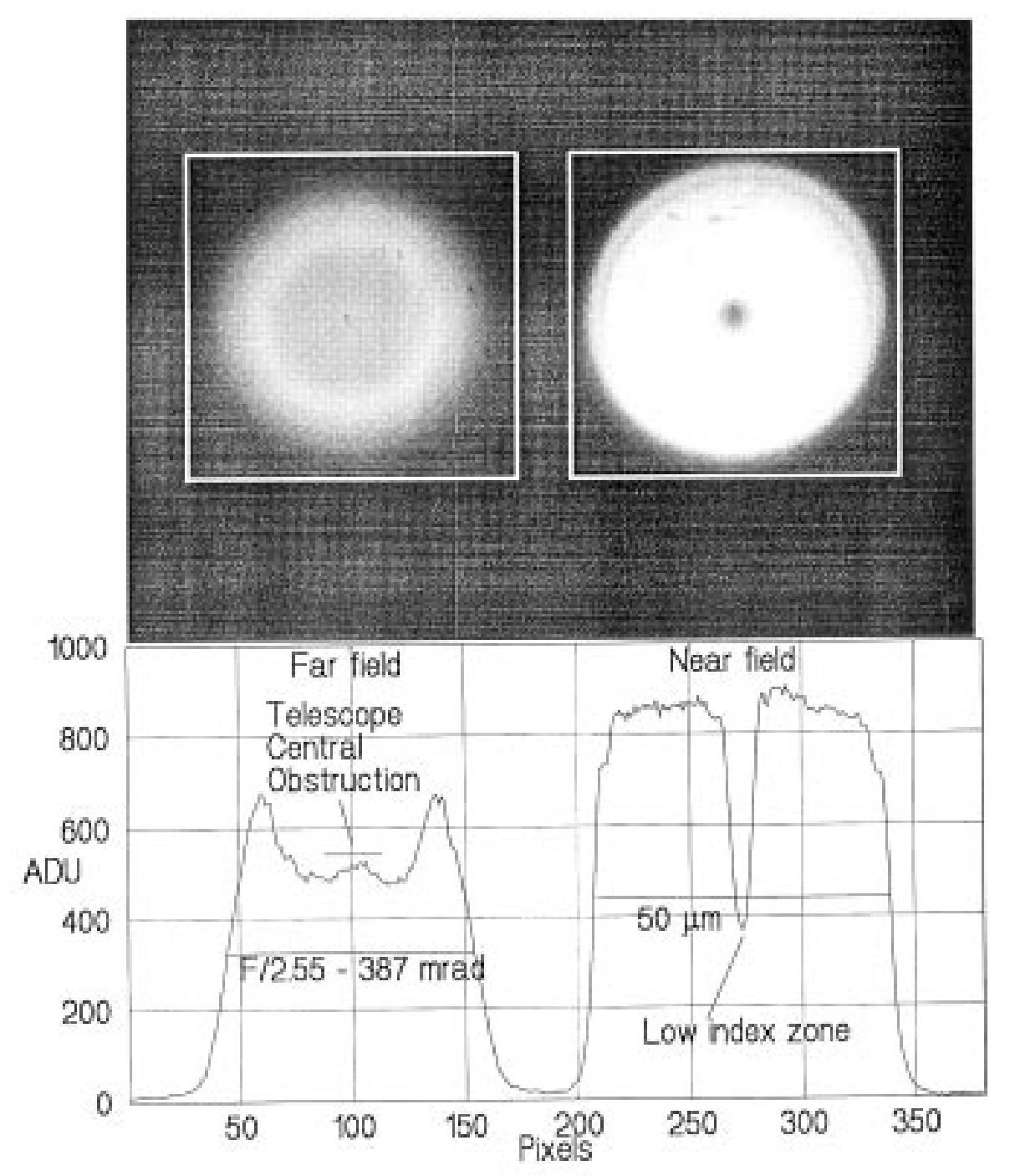

Fig. 13. Output beam cross-section with star (152-cm telescope, $50 \mu \mathrm{m}$ fiber). Near-field: left; far-field: right. Two windows $(160 \times 160$ pixels each $)$ used for computation are shown. $50 \mu \mathrm{m}$ and $\mathrm{f} / 2.55$ indicated dimensions are reduced to fiber output

be worthwhile. Altogether, it is safe to assume that the RV fluctuations from $M_{1}$, etc. may prove distincly larger than those just computed from $X_{1}$ and $Y_{1}$. We have seen that in the ELODIE case, the near-field fluctuations alone contributed to $\sim 4 \mathrm{~m} / \mathrm{s}$ RV change, whereas the measured radial velocity fluctuations reached $\sim 9 \mathrm{~m} / \mathrm{s}$. On the other hand, addition of a double scrambler should give some further improvement.

With the ELODIE $100-\mu \mathrm{m}$ fiber, we found (see Sect. 3.3.2) that the RMS fluctuations of the near-field were equal to $1 / 1800$ of the fiber diameter. This figure seems to confirm that the autoguider brings a gain of $\sim 3$ in the fluctuations of the stellar beam at the output of the fiber. Since our $50-\mu \mathrm{m}$ fiber reduces the star image motion by a factor 100 , this means that these motions are about $1 \mu \mathrm{m}$ RMS, or 0.05 arcsec, which is the RMS residual photocenter motion with FLAG active.
Table 1. RMS computed from a 3 orders polynomial fit for each the parameters of Figs. 14a and 14b

\begin{tabular}{rcc}
\hline & Hand-Guiding & FLAG. \\
\hline Near Field & & \\
INT1 $($ ADU $)$ & 60.0 & 33.7 \\
$X_{1}(\mathrm{~nm})$ & 43.9 & 9.53 \\
$Y_{1}(\mathrm{~nm})$ & 22.0 & 10.9 \\
$M_{1}(\mathrm{~nm})$ & 54.9 & 22.7 \\
\hline Far Field & & \\
$X_{2}(10 \mu \mathrm{rad})$ & 12.5 & 2.93 \\
$Y_{2}(10 \mu \mathrm{rad})$ & 12.0 & 5.65 \\
$M_{2}(100 \mu \mathrm{rad})$ & 16.7 & 7.0 \\
\hline
\end{tabular}

\section{Conclusion and possible extensions}

We have confirmed that the incomplete scrambling action of a fiber feeding a spectrograph leaves RV errors at a level 
of few $\mathrm{m} / \mathrm{s}$. We have described a fiber-locked autoguider specifically built to reduce the stellar-beam geometrical fluctuations within the spectrograph; these were reduced by a factor of $\sim 3$ even while instantaneous image blurring was not improved. While fluctuations do not vanish, the gain is appreciable, and the system is fully compatible with a future double scrambler. On the other hand, a mere attempt to make FLAG faster does not seem worthwhile: our 0.05 arcsec RMS residual photocenter-motion under 2.2-arcsec seeing, seems to imply that the corresponding RV errors from any spectrograph will be negligible compared with those arising from changes in image blurring; these might be corrected only by AO.

Presently FLAG needs 4 plane-parallel plates and is adapted to a f/27 Coudé beam, but we can imagine other configurations with better efficiency. If many 152 -cm telescope users at OHP were interested by a fiber link, FLAG could be adapted for the primary focus, which would eliminate 4 mirrors and reduce the telescope central obstruction. Also, the plane-parallel plates might be replaced by a single ellipsoïdal off-axis mirror; this would be supported by a PZT-driven tip-tilt platform and used both for scanning and guiding in $X$ and $Y$. The field of acquisition/correction would be small ( $\sim 2$ arcsec), but one might keep a pierced mirror as finder; very low-frequency guiding errors might be sent to the fine guiding telescope motors. The fiber input itself would oscillate in $Z$. The electronic control would be almost unchanged. A similar FLAG could also be built for the Cassegrain focus of larger telescopes.

Altogether, we make no claim to have solved the scrambling problem; we merely hope that our plain FLAG will help, as long as adaptive optics are unavailable.

Acknowledgements. We are grateful to the members of the Observatoire de Haute-Provence J.P. Sivan, D. Kohler, G. Adrianzyk, A. Moulet and G. Rau for their help. We thank J. Schmitt, M. Martic, J.C. Lebrun and F. Moulin for their help during ELODIE campaigns. The FLAG project was made possible thanks to the support received from the Institut National des Sciences de l'Univers and from the Programme National de Planétologie.

\section{References}

Baranne A., et al., 1996, A\&A 119, 373

Barden S.C., 1995, in: Fiber Optics in Astronomical Application, S.C. Barden (ed.), Proc. SPIE Vol. 2476, p. 2

Bouchy F., et al., 1999, in: Precise Stellar Radial Velocities, Hearnshaw J.B. and Scarfe C.D. (eds.), ASP Conf. Ser. (in press)

Brown T.M., 1990, in: CCDs in Astronomy, Jacoby G. (ed.), San Francisco, ASP, ASP Conf. Ser. 8, 335

Brown T.M., et al., 1991, ApJ 368, 599

Brown T.M., et al., 1994, PASP 106, 1285

Butler R.P., et al., 1996, PASP 108, 500

Casse M., 1995, thesis in: Conception d'un spectrographe multiobjets haute résolution pour le Very Large Telescope européen et études des performances de stabilité de mesure des vitesses radiales des couplages par fibres optiques, University of Paris XI

Close L.M., McCarthy D.W., 1994, PASP 106, 77

Cochran W.D., Hatzes A.P., 1994, Ap\&SS 212, 281

Connes P., 1985, Ap\&SS 110, 211

Connes P., et al., 1996, Ap\&SS 241, 61

Golimowski D.A., et al., 1992, Appl. Opt. 31, 4405

Heacox W.D., 1987, J. Opt. Soc. Am. A 4, 488

Heacox W.D., Connes P., 1992, A\&AR 3, 169

Hecquet J., Coupinot G., 1985, J. Opt. 16, 21

Hunter T.R., Ramsey L.W., 1992, PASP 104, 1244

Maaswinkel F., Bortoletto F., D'odorico S., Huster G., 1988, in: Instrumentation for Ground-Based Optical Astronomy, Present and Future, The Ninth Santa Cruz Summer Workshop in Astronomy and Astrophysics, Robinson L.B. (eds.)

Martin H.M., 1987, PASP 99, 1360

Mayor M., Queloz D., 1995, Nat 378, 355

Pickles A.J., Youhg T.T., Nakamura W., et al., 1994, in: Advanced Technology Optical Telescopes V, Stepp L.M. (ed.), Proc. SPIE 2199, 504

Racine R., McClure R.D., 1989, PASP 101, 731

Ramsey L.W., 1988, in: Fibers Optics in Astronomy, Barden S.C. (ed.). Tucson, ASP, ASP Conf. Ser. 3, 26

Schmitt J., 1997, thesis in: Étude et réalisation en laboratoire d'un accéléromètre astronomique absolu, University of Paris VI

Thompson L., Ryerson H., 1984, in: Instrumentation in Astronomy V, Boksenberg A., Crawford D.L. (eds.), Proc. SPIE 445, 560

Walker G.A.H., et al., 1995, Icarus 116, 359 